\title{
Ternary Diagram of Bithermal Systems
}

\author{
J. Ramousse* \\ Univ. Grenoble Alpes, Univ. Savoie Mont Blanc, CNRS, LOCIE, 73000 Chambéry, France \\ *E-mail: julien.ramousse@univ-smb.fr
}

Received 25 September 2017, Accepted 30 January 2018

\begin{abstract}
This paper proposes an original and synthetic graphical representation of bithermal systems operation on a normed ternary diagram $\left(q_{h}, q_{c}, w\right)$. Thanks to the normed axes, an intuitive graphical interpretation of the operating conditions is derived by using polar coordinates. The energy flow intensity involved in the system is directly linked to its distance $r_{M}$ to the origin and its efficiency is only related to the angle $\alpha$ defined in this work. Thus, the potential operating modes depending on the energy flow directions, are distributed into sectors of angle $\pi / 3$. In addition to the potentially reversible operating modes (heat engine and heat pump modes), the two dissipative operating modes (forced heat transfer and thermal dissipation modes) are also described. Moreover, the characterization of the operating mode interfaces validates the physical continuity of the proposed description. According to the second law of thermodynamics, the operation of bithermal systems is restricted to the top half-plane bounded by the Carnot boundary (function of the reservoirs temperature ratio). Furthermore, the introduction of an unconventional definition of the energy efficiency when the hot reservoir is used as a heat sink leads to positive and below unity efficiencies in both reversible modes and negative efficiencies in both dissipative modes. In order to illustrate the use of the proposed representation, two examples are introduced: (i) operation of the classical thermodynamics cycles of Carnot, Stirling and Erricson is plotted for graphical interpretation, (ii) endoreversible (exo-irreversible) system representation helps to rediscover graphically the Chambadal/Novikov/Curzon-Ahlborn efficiency (constant energy efficiency at maximum work in heat engine mode).
\end{abstract}

\section{Keywords: Ternary diagram; polar coordinates; bithermal system operating modes; energy conversion efficiency.}

\section{Introduction}

Heat energy conversion into work energy (and inversely) is of high interest in engineering because it serves widely used applications (heat engines, heating and cooling devices...). In response to this challenge, the science of thermodynamics emerged in the nineteenth century [1-3] for the study of work-heat energy conversion systems. Bithermal systems are classically considered to describe machines allowing such energy conversions, as two distinct temperatures are needed to convert heat into work. A bithermal system is thus defined as a thermodynamic system operating in cycles or steady state exchanging heat with two distinct heat reservoirs at different temperature. As a rule, bithermal system operation is governed by the first law of thermodynamics, that links the energy interactions between the system and its environment, and the second law, that expresses the efficiency of the work-heat energy conversion.

In order to ease the description and the interpretation of bithermal systems, several graphical tools have been suggested. Thermodynamic cycles are classically represented on 2D phase diagrams that plot the equilibrium states of the working fluid involved in the cycle depending on two independent thermodynamic properties (pressure $P$, temperature $T$, specific volume $v$, specific enthalpy $h$, specific entropy $s$ ). The most frequently used phase diagrams are the $P-v$ Clapeyron diagram [4], the $h$-s Mollier diagram [5] and the $T-s$ temperature-entropy diagram. These diagrams allow graphical description of thermodynamic cycles (Carnot, Stirling, Erricson, Rankine and others) by means of complementary transformations (see [6-10] for instance). The work and heat energy flows involved in each transformation of the cycle are computed with the help of thermodynamic relationships. The operating mode of the system is deduced from the sense of the cycle is operated (clockwise in heat engine mode or anti-clockwise for heat pump mode). Although these representations are highly useful for the description of thermodynamic cycles, further analyses are needed to evaluate the energy flows balance over the cycle and determine the corresponding energy conversion efficiency.

On the other hand, more systemic representation has been proposed by means of the energy flows exchanged over a cycle by bithermal systems, thus focusing on their operating mode and efficiency. Through the literature, the following three main representations deserve a particular attention. Bejan [6] identified the wedge of minimum- $Q$ in a temperature-energy diagram (also called the $T-Q$ graphic method) by considering ideal (reversible) operation. As real (non-reversible) operation implies an increase of the heat released to the heat sink, its operation is deduced from the resulting deviation from reversible operation. It is worthy of note that the entropy generation (related to the irreversibilities) can be linked to the cotangent of the deviation. However, this representation only covers the two potentially reversible operating modes (heat engine and heat pump modes) by means of two distinct but similar representations as the heat energy flows are counted in absolute values. 
Another interesting graphical representation was proposed by Borel and Favrat in [8]. The authors suggested plotting bithermal systems on a graph with the reservoirs temperature ratio and the work-heat energy ratio as orthogonal axes. The energy and exergy efficiencies were then extended to $3 \mathrm{D}$ plots. The different operating modes of bithermal systems (heat engine mode, heating and cooling heat pump modes and dissipative modes) were distinguished, but the two dissipative modes identified have not been qualified and discussed in details. Moreover, assuming that one heat reservoir is at ambient temperature (the ambient could either be the hot or the cold reservoir) introduces some operating mode discontinuities that are somehow confusing and vanish in the proposed representation.

The last notable graphical representation was proposed by Raveau [9-11] where bithermal system operation is plotted with the hot and cold heat flows $\left(Q_{h}\right.$ and $\left.Q_{c}\right)$ as orthogonal axes. Alefeld and Radermarcher generalized this graphical approach to multi-heat reservoirs in [12]. Although the work exchanged can be deduced on the bisector of the $Q_{h}$ and $Q_{c}$ axes, the $W$-axis is not normed. Consequently, the use of polar coordinates is not adapted for further graphical interpretation. Even though the suggested representation introduced in [13] and detailed in this paper is close to that last one, significant improvements are obtained with the use of the proposed normed ternary diagram $\left(q_{h}, q_{c}, w\right)$ and the introduction of polar coordinates. Mainly, the energy conversion efficiency is directly linked to the geometric angle introduced later in this work. This work thus aims to complement the above graphical representations for further understanding and easier graphical interpretation of bithermal systems operation.

The first sections are dedicated to introduce the proposed representation of bithermal systems operation in a ternary diagram with a particular attention to highlight the differences with the previous studies. System energy conversion efficiency is discussed in section 4 , leading to introduce a non-conventional energy efficiency definition when the hot heat reservoir is used as a sink. The benefit for graphical interpretation of the proposed normed ternary diagram is then discussed in section 5, with the introduction of polar coordinates. Finally, the paper concludes with two illustrative examples: (i) the exoreversible thermodynamic cycles of Carnot, Stirling and Erricson are plotted for a complete analysis and comparison of their operating conditions; (ii) endoreversible (exo-irreversible) cycles are plotted to rediscover graphically the energy efficiency of Novikov, Chambadal and Curzon-Ahlborn [14-16] at maximum work in heat engine mode.

\section{Bithermal Systems}

\subsection{Definitions and Convention}

Let us consider a bithermal system, as presented in Figure 1 , exchanging heat energies with two heat reservoirs at different temperatures. Since a temperature difference is needed to convert heat into work, the reservoirs can be distinguished with respect to their relative thermal potential, so that the hot reservoir temperature is $T_{h}$ and the cold one is $T_{c}$, with $T_{h}>T_{c}$. Thermal reservoirs are assumed to be infinitely large compared to the amount of heat given to or released by the system $\left(q_{h}\right.$ and $q_{c}$ ), so that their respective temperatures $T_{h}$ and $T_{c}$ remain constant. To generalize the proposed approach to bithermal systems operating in cycles or steady state, energy and entropy balances are written on a unit of mass involved in the process, thus considering the specific energy and specific entropy. Such systems are classically described using the first and second laws of thermodynamics, as detailed in the following.

In order to keep the proposed description consistent, heat and work energy flows are counted algebraically according to the conventional approach in mechanics, i.e., positive when entering the system and negative when leaving the system (see Figure 1). The thermodynamic system considered could either work as a generator when work is extracted from the system $(w<0)$ or as a receptor when work has to be fed to the system $(w>0)$. Similarly, the reservoir operates as a heat source when heat is transferred from the reservoir to the system $(q>0)$ or as a heat sink when heat is released from the system to the reservoir $(q<$ $0)$.

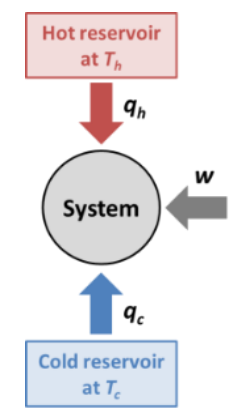

Figure 1. Bithermal thermodynamic system representation (Figure is in color in the on-line version of the paper).

\subsection{First Law of Thermodynamics}

The first law of thermodynamics applied to a unit of mass involved in bithermal systems operating in cycles or steady state writes:

$q_{h}+q_{c}+w=0$

As this equation is representative of a plane equation, any bithermal system $\mathrm{M}\left(q_{h}, q_{c}, w\right)$ can be plotted in the corresponding geometrical plane $P$. This paper proposes to identify this plane $P$ for easier representation and interpretation of bithermal systems.

\subsection{Second Law of Tthermodynamics}

The second law of thermodynamics applied to a unit of mass involved in bithermal systems operating in cycles or steady state writes:

$\frac{q_{h}}{T_{h}}+\frac{q_{c}}{T_{c}}+\sigma=0$

where $\sigma$ is the specific entropy generation in the system, defined positive $\sigma \geq 0$. This term is related to the irreversibilities occurring in the system.

The second law of thermodynamics thus reduces the operating conditions reachable by bithermal systems, as it implies some impossible transformations $(\sigma<0)$. Consequently, the realistic operating conditions of bithermal systems only cover a part of the plane $\mathrm{P}$, as detailed in the following.

\section{Graphical Representation \\ 3.1 Introduction to Ternary Diagram}

Any bithermal system $\mathrm{M}\left(q_{h}, q_{c}, w\right)$ can be plotted in the geometric plane $P$ defined by Eq. (1) (perpendicular to the trisectrice of the $3 \mathrm{D}$ orthonormal axes $q_{h}{ }^{\prime}, q_{c}{ }^{\prime}$ and $\left.w^{\prime}\right)$. The 
(non-orthogonal) normed axes $q_{h}, q c$ and $w$ presented in Figure 2 are deduced from projection of the 3D orthonormal axes $q_{h}{ }^{\prime}, q_{c}{ }^{\prime}$ and $w^{\prime}$ in the plane $P$. Consequently, the normed axes $q_{h}, q c$ and $w$ are linked by a $2 \pi / 3$ in-plane rotation with $M^{\circ}(0,0,0)$ as center of rotation. This representation allows plotting any bithermal thermodynamic system from the knowledge of only two of the three energies $q_{h}, q c$ and $w$ exchanged between the system and its surroundings. The missing data is directly read on the diagram.

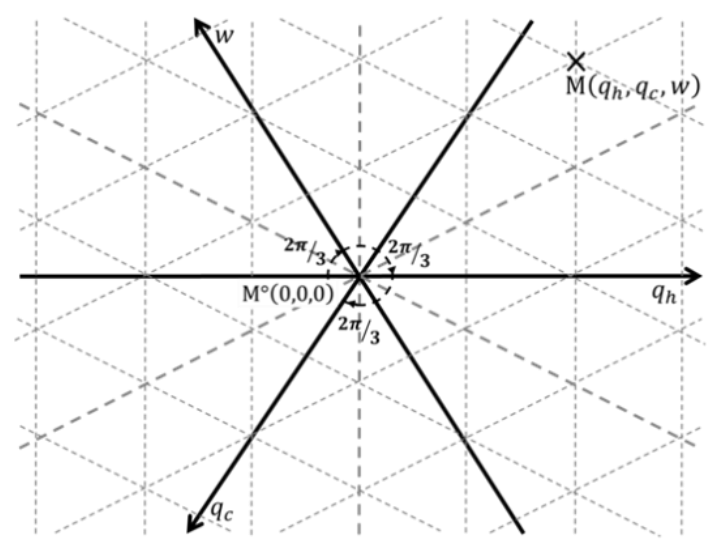

Figure 2. Ternary diagram of bithermal systems.

The representation proposed is closed to that introduced by Raveau (see [9-11] for instance), where bithermal systems are plotted on a graph defined with the orthonormal axes $q_{h}$ and $q_{c}$. In that case, the work exchanged by the system $w$ could be read on the bisector line of the axes $q_{h}$ and $q_{c}$. However this axis is not normed, in contrary to the normed axis $w$ introduced in the proposed ternary diagram. Even if the work exchanged by the system can be easily deduced numerically thanks to Eq. (1), it is relevant to retrieve it graphically for a complete graphical interpretation.

\subsection{Operating Modes}

As shown in Figure 3, six different regions (sextants) can be distinguished on plane $P$, depending on the sign of the energy flows $q_{h}, q c$ and $w$. To each region corresponds a different operating mode, as detailed in the following. The region numbering is arbitrary and is only given for interpretation purposes.

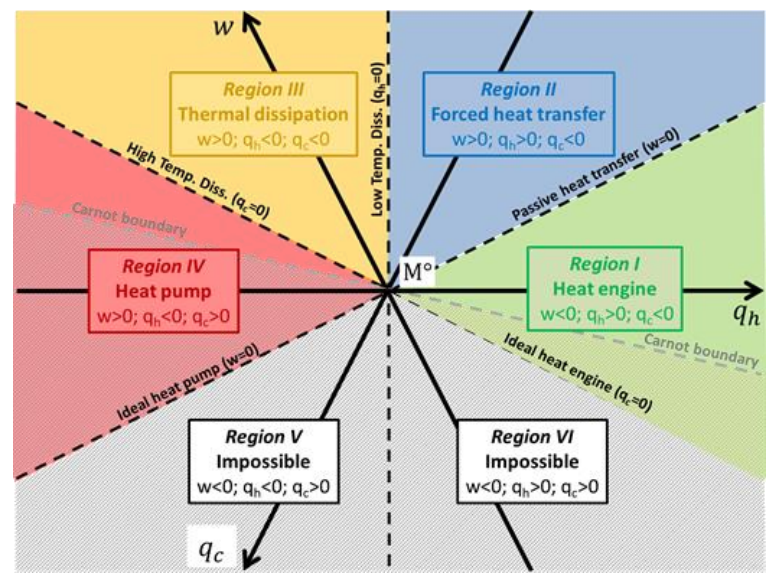

Figure 3. Operating mode representation in plane $P$. The Carnot boundary is plotted for $\theta=1 / 3$ (Figure is in color in the on-line version of the paper).

\section{- Region I: Heat engine}

$w<0, q_{h}>0, q_{c}<0$

This mode is highly advantageous for scientists because it is the only mode in which the system works as a generator $(w<0)$. In this mode the system produces work from heat transfer from the high-temperature heat source to the lowtemperature heat sink. In other words, the system converts a part of high thermal exergy taken from the hot heat source into work exergy and low thermal exergy released to the cold heat sink.

- Region II: Forced heat transfer $w>0, q_{h}>0, q_{c}<0$

When operating in this mode, the system uses the work received to intensify the heat transfer from the hot heat source to the cold heat sink. This mode is currently used in heat exchangers in forced convection: heat transfer is increased thanks to the work given to the system to make up for the pressure drops of the flowing fluids. From the exergy point of view, work exergy and high thermal exergy from the hot heat source are dissipated to low thermal exergy at the cold heat sink.

- Region III: Thermal dissipation $w>0, q_{h}<0, q_{c}<0$

In this operating mode, the system converts the work energy received into thermal energy to the hot and the cold heat sinks. No heat source is connected to the system. The work exergy transferred to the system is fully dissipated as low and high thermal exergies to the heat sinks.

If the hot and cold heat sinks are thermally connected, bithermal systems operating in this mode could be assimilated to monothermal systems exchanging heat $q_{m}$ with a reservoir at mean temperature $T_{m}$, so that:

$q_{m}=q_{h}+q_{c}=-w \leq 0$ and $\frac{q_{m}}{T_{m}}=\frac{q_{h}}{T_{h}}+\frac{q_{c}}{T_{c}}=-\sigma$

Given that the heat energy flows exchanged $q_{h}, q_{c}$, and $q_{m}$ are all negative, the equivalent mean temperature is bounded by the hot and cold heat sink temperatures: $T_{h} \geq T_{m} \geq T_{c}$.

\section{- Region IV: Heat pump $\quad \mathrm{w}>0, \mathrm{q}_{\mathrm{h}}<0, \mathrm{q}_{\mathrm{c}}>0$}

In this mode, the system runs as a receptor to transfer heat from the low-temperature heat source to the hot heat sink thanks to the work received. In terms of exergy, work exergy is used to transfer thermal exergy from a low thermal level to a higher thermal level. Systems operating in this mode are most of the time qualified of either heat pump or refrigerator depending on the useful effect expected (heating or cooling), even though both effects occur simultaneously.

\section{- Region V: Impossible $\quad \mathrm{w}<0, \mathrm{q}_{\mathrm{h}}<0, \mathrm{q}_{\mathrm{c}}>0$}

According to the second law (Eq. (2)), this region is not attainable because the reservoirs temperature are distinct and positive $\left(T_{h} \geq T_{c} \geq 0\right)$. Indeed, heat cannot spontaneously flow from the cold heat source to the hot heat sink without external work supplied to the system.

- Region VI: Impossible $\quad w<0, q_{h}>0, q_{c}>0$

Once again, this region is not attainable according to the second law (Eq. (2)), since the reservoirs temperature are positive $\left(T_{h} \geq T_{c} \geq 0\right)$. Indeed, a cold heat sink is needed to convert heat energy from a hot heat source into work energy.

Note that, in accordance with Eq. (1), no region corresponds to the following operating conditions (not included in plane $P$ ):

$$
\begin{aligned}
& \text { - } \quad w>0, q_{h}>0, q_{c}>0 \\
& \text { - } \quad w<0, q_{h}<0, q_{c}<0
\end{aligned}
$$

Consequently, the realistic operating modes of bithermal systems reduce to regions I-IV. In addition to the potentially reversible operating modes (heat engine and heat pump modes) mostly studied, two dissipative operating modes can be encountered: forced heat transfer (Region II) and thermal 
dissipation (Region III) modes. The operating mode of any bithermal system $\mathrm{M}\left(q_{h}, q_{c}, w\right)$ is thus directly deduced from its location in plane $\mathrm{P}$ with respect to the above-detailed regions.

\subsection{Operating Modes Continuity}

The continuity between the different operating modes of the proposed representation appears naturally in Figure 3. Operating mode interfaces are therefore qualified as follows:

- Region VI/Region I interface: This condition is reached when $q_{c}=0$ and $w=-q_{h}$, meaning that the thermal energy supplied by the hot heat source is fully converted into work energy. By passage to the limit, this operating condition can only be reached by an ideal ${ }^{1}$ heat engine.

- Region I/Region II interface: This interface is reached when $w=0$, meaning $q_{h}=-q_{c}$. This operating condition is interpreted as passive heat transfer (conduction, radiation and free convection).

- Region II/Region III interface: On this interface, $q_{h}=$ 0 , leading to $w=-q_{c}$. The work energy is fully transferred as thermal energy to the cold heat sink. This operating condition corresponds to low-temperature thermal dissipation.

- Region III/Region IV interface: In this case, $q_{c}=0$ and $w=-q_{h}$. The work energy supplied to the system is thus fully dissipated to the hot heat sink, resulting in hightemperature thermal dissipation.

- Region IV/Region V interface: As for the Region I/Region II interface, this can be interpreted as passive heat transfer $\left(w=0\right.$ and $q_{h}=-q_{c}$,). By passage to the limit, it can also be understood as an ideal ${ }^{2}$ heat pump as the work supplied is null.

At the origin $\mathbf{M}^{\circ}\left(q_{h}=q_{c}=w=0\right)$, no energy flows through the system: the system is in thermodynamic equilibrium.

\subsection{Reversible Carnot Boundary}

Note that the graphical representation proposed in the above sections is kept valid regardless the reservoirs temperature. However, the second law of thermodynamics (Eq. (2)) restricts more the realistic operating conditions reachable by the bithermal system depending on the reservoirs' thermal quality. Indeed, as the specific entropy generation is defined positive, Eq. (2) leads to the following inequality:

$\frac{q_{h}}{T_{h}}+\frac{q_{c}}{T_{C}} \leq 0$

Hence, depending on the hot reservoir is a hot heat source or a hot heat sink, the thermal energy ratio exchanged by the system is bounded by the reservoirs temperature ratio $\theta=$ $T_{c} / T_{h}$

$\begin{array}{lll}\text { - } & \text { Hot heat source }\left(q_{h}>0\right): & \frac{q_{c}}{q_{h}} \leq-\theta \\ \text { - Hot heat } \operatorname{sink}\left(q_{h}<0\right): & \frac{q_{c}}{q_{h}} \geq-\theta\end{array}$

In the case of a reversible Carnot system $(\sigma=0)$, the above inequalities turns into the following equality, defining the Carnot boundary:

\footnotetext{
1 The term "ideal" refers to a reversible system $(\sigma=0)$ working with ideal reservoirs temperature, i.e., $T_{h} \rightarrow+\infty$ and $T_{c}=0(\theta=$ $0)$ in heat engine mode and $T_{h}=T_{c}(\theta=1)$ in heat pump mode.
}

$\frac{q_{c}{ }^{C}}{q_{h}{ }^{C}}=-\frac{T_{C}}{T_{h}}=-\theta$

with $0 \leq \theta \leq 1$.

Graphically, the Carnot boundary results in a straight line passing through the origin $\mathrm{M}^{\circ}(0,0,0)$ whose slope is a function of the reservoirs temperature ratio $\theta$ (see Figure 3 ). The slope is expressed in the following as a function of the angle $\alpha$ introduced in section 5.2. According to the inequalities given in Eq. (4), bithermal systems can only be plotted on the top half-plane bounded by the Carnot boundary.

Since the reservoirs temperature are defined positive $\left(T_{h} \geq T_{c} \geq 0\right.$, i.e. $\left.0 \leq \theta \leq 1\right)$, the Carnot boundary only plots in reversible modes (Regions I and IV), where $-1 \leq$ $q_{c} / q_{h} \leq 0$. As Regions II and III are related to dissipative operating modes, they cannot be described by an equivalent reversible Carnot system, otherwise it would imply $\theta>1$ in Region II $\left(q_{c} / q_{h} \leq-1\right)$ and $\theta<0$ in Region III $\left(q_{c} / q_{h} \geq\right.$ $0)$.

\subsection{Comparison to Graphical Representations of the Literature}

Based on reversible cycle considerations, the wedge of minimum heat exchanged in heat engine and heat pump modes (potentially reversible operating modes) can be plotted as a function of the reservoir temperatures in a $Q-T$ diagram [6]. The corresponding reversible work energy (available in heat engine mode or needed in heat pump mode) is deduced from the reversible heat energies difference. Nonreversible operation implies an increase of the heat energy released to the heat sink (either to the cold reservoir in case of heat engine operation or to the hot reservoir in case of heat pump operation). The work lost (i.e. decrease of the work available in heat engine mode or increase of the work needed in heat pump mode) could then be deduced from the deviation from the reversible operation. However, the operating mode considered, and thus the corresponding energy flow directions (given to or released by the system), have to be known a priori as the energy flows are plotted in absolute values. Consequently, this approach is restricted to the two potentially reversible operating modes (heat engine and heat pump modes) with distinct analysis. In contrast, the graphical representation introduced in this paper allows a general graphical analysis for all possible operating modes, including the two dissipative modes (forced heat transfer and thermal dissipation).

Otherwise, the different operating modes of bithermal systems (i.e. heat engine mode, heating and cooling heat pump modes, dissipative modes and impossible operating modes) are highlighted in the adimensional representation suggested by Borel and Favrat [8]. However, the two dissipative operating modes have not been distinguished and discussed in detail. Furthermore, assuming that one heat reservoir is at ambient temperature $T_{a}$ (meaning the ambient could either be the hot or the cold reservoir) leads to the following main differences from the proposed representation:

- The single heat engine operating mode (Region I) is distributed in two distinct and non-adjacent regions, 
depending on the reservoirs temperature $\left(T_{a}=T_{h}>T_{c}\right.$ or $T_{h}>T_{c}=T_{a}$ ).

- Similarly, the single heat pump mode (heating and cooling) is divided into two distinct but adjacent regions. Heating and cooling modes are distinguished with respect to the ambient temperature that could either play the role of a hot heat sink (cooling mode with $T_{a}=T_{h}>T_{c}$ ) or a cold heat source (heating mode with $T_{h}>T_{c}=T_{a}$ ).

These discontinuities are somehow confusing and vanish in the proposed representation. However, the use of the reservoirs temperature ratio as ordinate axis sets a permanent reversible Carnot boundary limit, unlike the proposed representation that needs the slope of the Carnot boundary to be determined as a function of the reservoirs temperature.

The proposed representation is close to the one introduced by Raveau in [9-11], where the bithermal systems are plotted on a graph defined with the orthonormal basis $q_{h}$ and $q_{c}$. Although the work exchanged can be deduced on the bisector of the $q_{h}$ and $q_{c}$ axes, the $w$-axis is not normed. In contrast, the ternary diagram proposed in this study relies on the normed axes $\left(q_{h}, q_{c}\right.$ and $\left.w\right)$ that promote the introduction of polar coordinates for further graphical interpretation. As a consequence of the modified axes, the identified regions corresponding to the different operating modes are slightly altered. On the other hand, the energy conversion efficiency could be directly linked to the geometric angle introduced in the following. The interpretation of the different operating modes is thus improved, particularly for the two dissipative modes not thoroughly described.

This paper thus aims to complement the above graphical representations for further understanding and easier graphical interpretation.

\section{System Efficiency}

To analyze the bithermal systems performance, this section discusses the system energy efficiencies, depending on the hot reservoir is whether used as a heat source $\left(q_{h}>\right.$ 0 ) or a heat sink $\left(q_{h}<0\right)$.

\subsection{Hot Heat Source}

With respect to the classical heat engine efficiency definition, the following expression is used when the hot reservoir is a heat source $\left(q_{h}>0\right)$ :

$\eta_{q_{h^{+}}}=\frac{-w}{q_{h}}=1-\theta-\frac{\sigma T_{c}}{q_{h}}$

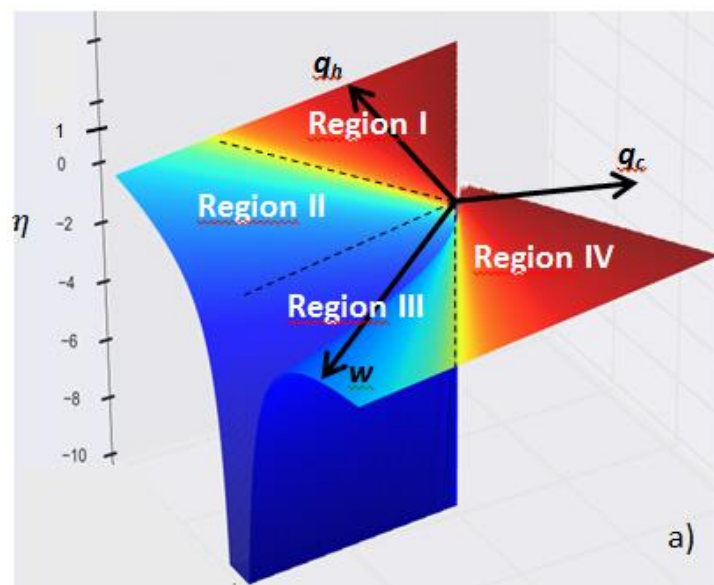

Consequently, any bithermal system running in Region I $\left(w<0, q_{h}>0, q_{c}<0\right)$ shows a positive and below-unity energy efficiency. The energy efficiency reaches unity for an ideal heat engine (Region VI/Region I interface) and decreases continuously to zero for passive thermal transfer (Region I/Region II interface). Then, systems running in Region II $\left(w>0, q_{h}>0, q_{c}<0\right)$ show negative energy efficiency, as expected for this dissipative operating mode. It decreases from zero for passive thermal transfer (Region I/Region II interface) to negative infinity for lowtemperature thermal dissipation (Region II/Region III interface).

For reversible operation, the Carnot efficiency ( $\sigma=0)$ of a system coupled to a hot heat source is given by:

$\eta_{q_{h^{+}}{ }^{C}}=1-\frac{T_{C}}{T_{h}}=1-\theta$

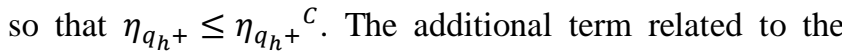
entropy generation in Eq. (6) thus decreases the energy conversion efficiency as it expresses the deviation from reversible operation $\left(\frac{\sigma T_{C}}{q_{h}}=-\frac{q_{c}}{q_{h}}-\frac{T_{c}}{T_{h}}\right)$.

\subsection{Hot Heat Sink}

Considering a system $\Sigma^{H P}$ operating in heat pump mode $\left(w^{H P}>0, \quad q_{h}{ }^{H P}<0, \quad q_{c}{ }^{H P}>0\right)$, the energy flows exchanged with its surroundings can be counterbalanced by a complementary system $\Sigma^{H E}$ operating in heat engine mode, so that: $w^{H E}=-w^{H P}<0, q_{h}{ }^{H E}=-q_{h}{ }^{H P}>0$ and $q_{c}{ }^{H E}=$ $-q_{c}{ }^{H P}<0$. These two complementary systems do not need external work and both heat reservoirs do not exchange any thermal energy. It therefore appears natural to define the energy efficiency of heat pump systems as the complement to unity to the heat engine energy efficiency. The energy conversion efficiency for a system coupled to a hot heat sink $\left(q_{h}<0\right)$ could thus be written:

$\eta_{q_{h^{-}}}=1-\eta_{q_{h^{+}}}=\frac{q_{c}}{-q_{h}}=\theta+\frac{\sigma T_{c}}{q_{h}}$

This coefficient could be interpreted as the energy efficiency of the system to extract heat from the cold source and transfer it to the hot heat sink. It remains positive and below unity in heat pump mode (Region IV: $\mathrm{w}>0, \mathrm{q}_{\mathrm{h}}<0$, $\mathrm{q}_{\mathrm{c}}>0$ ). It reaches unity for an ideal heat pump (Region $\mathrm{VI} /$ Region $\mathrm{V}$ interface) and decreases continuously to zero for high-temperature thermal dissipation (Region III/Region IV interface). Then, systems running in thermal dissipation

Figure 4.a/ 3D plot of the energy efficiency of bithermal energy conversion systems. $b / 2 D$ projection (Figure is in color in the on-line version of the paper). 
mode (Region III: $w>0, q_{h}<0, q_{c}<0$ ) have negative energy efficiency, decreasing from zero for high-temperature thermal dissipation (Region III/Region IV interface) to negative infinity for low-temperature thermal dissipation (Region II/Region III interface).

For reversible operation, the Carnot efficiency $(\sigma=0)$ of a system coupled to a hot heat sink is given by:

$\eta_{q_{h^{-}}{ }^{C}}=\frac{\operatorname{COP}^{C}{ }_{c}}{\operatorname{COP}^{C}}=\frac{T_{C}}{T_{h}}=\theta$

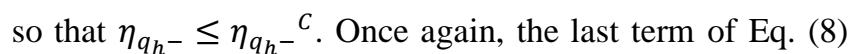
related to the entropy generation decreases the energy conversion efficiency (as $q_{h}<0$ ) by meaning the deviation from reversible operation.

\subsection{Graphical Representation}

According to the above definitions, the energy efficiencies are plotted as a 3D plot on the ternary diagram proposed in Figure 4a. Projections in plane $P$ are shown in Figures $4 \mathrm{~b}$ and 5 for easier interpretation. The energy efficiency definitions proposed leads to mirror symmetry whose axis of symmetry is perpendicular to axis $q_{h}$.

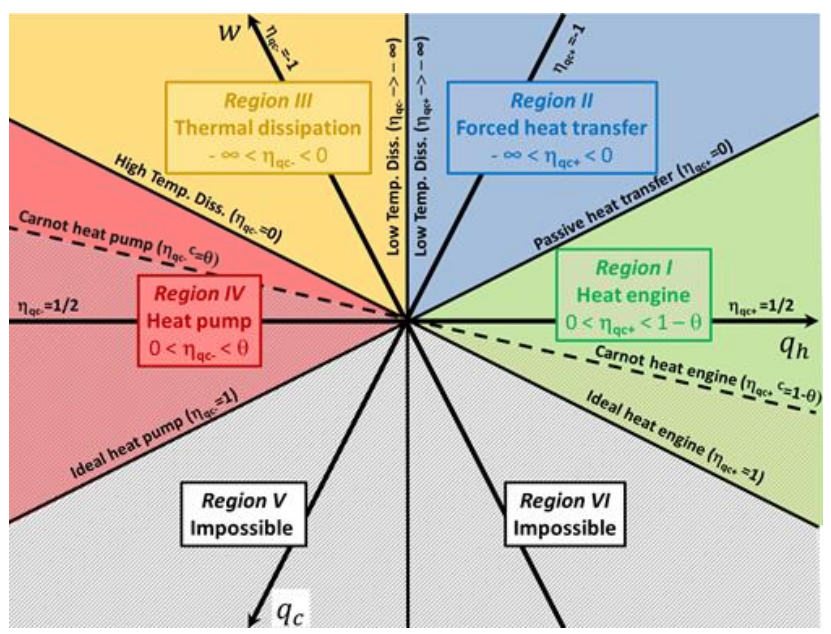

Figure 5. Schematic 2D plot of the energy efficiency of bithermal energy conversion systems in plane P (Figure is in color in the on-line version of the paper).

Among the above mentioned representations from literature, only the graphical illustration of Borel and Favrat [8] aims at the analysis of the energy conversion efficiency. Although this representation is close to the proposed 3D plot, they mainly differ from the energy efficiency definitions (in addition to the operating mode repartition discussed above). Indeed, the use of the conventional COP definitions in heat pump mode leads to energy efficiency values greater than unity. Furthermore, the energy efficiencies of the dissipative modes are not thoroughly discussed in [8] as they are just set to zero. With the unconventional energy efficiency definitions proposed herein, this coefficient remains bounded by unity in reversible operating modes and decreases continuously up to negative values in dissipative operating modes (Regions II and III). Hence, the above mentioned discontinuities vanish in the proposed representation.

\section{Graphical Interpretation Using Polar Coordinates}

Thanks to the normed axes, the system $\mathrm{M}\left(q_{h}, q_{c}, w\right)$ operating condition can be easily described using polar coordinates, with the radial distance $r_{M}$ to the origin $\mathrm{M}^{\circ}(0,0,0)$ and the angle $\alpha$ formed by the perpendicular to the axis $q_{h}$ and the point $\mathrm{M}\left(q_{h}, q_{c}, w\right)$ of vertex $\mathrm{M}^{\circ}(0,0,0)$, as introduced in Figure 6.

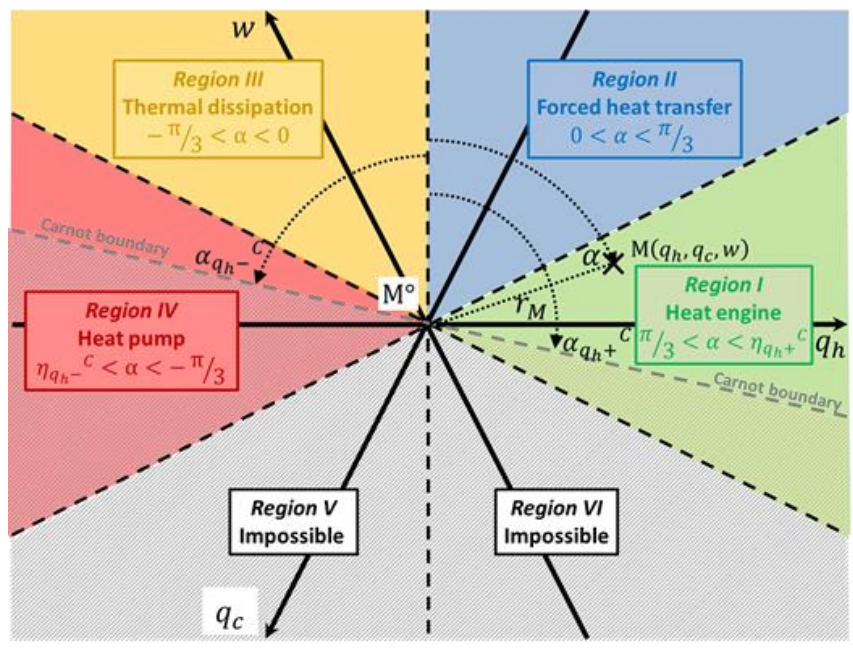

Figure 6. Interpretation of bithermal thermodynamic systems using polar coordinates (Figure is in color in the online version of the paper).

\subsection{Radius $r_{M}$}

According to trigonometric analysis, the radius $r_{M}$ of a bithermal system $\mathrm{M}\left(q_{h}, q_{c}, w\right)$ is given by:

$r_{M}^{2}=q_{h}{ }^{2}+\left(\frac{q_{c}-w}{\sqrt{3}}\right)^{2}=q_{c}{ }^{2}+\left(\frac{w-q_{h}}{\sqrt{3}}\right)^{2}=w^{2}+\left(\frac{q_{h}-q_{c}}{\sqrt{3}}\right)^{2}$

Whatever the operating mode considered, the specific energy flows can be distinguished with regards to their sign (i.e. inlet when positive and outlet when negative). The radius can thus be expressed in a general way as the quadratic sum of the inlet and outlet energy flows involved in the system:

$r_{M}^{2}=e_{\text {in }}^{2}+e_{\text {out }}^{2}$

where $e_{\text {in/out }}$ equals either the single energy flow involved in the direction considered or the energy flows difference divided by $\sqrt{3}$ when two energy flows are involved in the same direction. The $\sqrt{3}$ coefficient comes from the $2 \pi / 3$ angle between the energy axes. Therefore, the radius is linked to the energy intensity required for the bithermal system operation. The higher the radius is, the greater the energy amounts exchanged by the system.

With respect to the second law of thermodynamics (Eq. (2)), the radius could also be written as a function of the hot heat flow, the reservoirs temperature ratio and the entropy generation:

$r_{M}{ }^{2}=\frac{4}{3} q_{h}{ }^{2}\left[1-\theta+\theta^{2}-\frac{T_{c} \sigma}{q_{h}}\left(1-2 \theta-\frac{T_{c} \sigma}{q_{h}}\right)\right]$

As the irreversibilities influence the energy flows exchanged by the system, the corresponding radius could either increase or decrease depending on how the operating conditions evolve.

For reversible Carnot operation $(\sigma=0)$, the radius expression reduces to:

$r_{M}{ }^{2}=\frac{4}{3} q_{h}^{2}\left(1-\theta+\theta^{2}\right)$ 


\subsection{Angle $\alpha$}

Based on the angle $\alpha$ introduced above, classical trigonometric analysis leads to:

$\sin \alpha=\frac{q_{h}}{r_{M}}$ and $\cos \alpha=\frac{w-q_{c}}{\sqrt{3} r_{M}}$

The operating mode of the system considered is thus directly deduced from the angle $\alpha$ :

- $\alpha \in[\pi / 3 ; 2 \pi / 3]$ - Region I - Heat engine mode

- $\alpha \in[0 ; \pi / 3]$ - Region II - Forced heat transfer mode

- $\alpha \in[-\pi / 3 ; 0]$ - Region III - Thermal dissipation mode

- $\alpha \in[-2 \pi / 3 ;-\pi / 3]$ - Region IV - Heat pump mode

Furthermore, analytical development leads to the following expression:

$\tan |\alpha|=\frac{\sqrt{3}}{1-2 \eta_{i}}$

With $\eta_{i}=\eta_{q_{h^{+}}}$for $\alpha \in[0 ; 2 \pi / 3]$ and $\eta_{i}=\eta_{q_{h^{-}}}$for $\alpha \in$ $[-2 \pi / 3 ; 0]$.

This expression shows that the angle $\alpha$ only depends on the system energy efficiency $\left(\eta_{q_{h^{+}}}\right.$or $\left.\eta_{q_{h^{-}}}\right)$and highlights the mirror symmetry mentioned previously. It also expresses as a function of the specific entropy generation $\sigma$ and the reservoirs temperature ratio $\theta$ as:

$\tan \alpha=\frac{\sqrt{3}}{2 \theta-1+2 \frac{\sigma T_{c}}{q_{h}}}$

For Carnot systems $(\sigma=0)$, the maximum angle $\alpha^{C}$ is given by:

$\tan \alpha^{C}=\frac{\sqrt{3}}{2 \theta-1}$

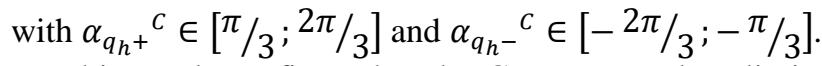

This result confirms that the Carnot Boundary limit is represented as a line whose slope is set by the reservoirs

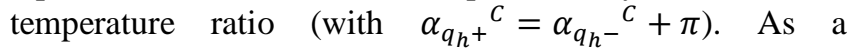
consequence, any real bithermal system must verify the following condition: $|\alpha| \leq\left|\alpha^{C}\right|$ and the entropy generation is directly linked to difference between the angles $\alpha$ and $\alpha^{C}$, as:

$\frac{\sigma T_{C}}{q_{h}}=\frac{\sqrt{3}}{2}\left(\frac{1}{\tan \alpha}-\frac{1}{\tan \alpha^{C}}\right)$

This result is consistent with the T-Q graphic method [6] where the entropy generation is linked to the cotangent of the deviation between the heat involved in non-reversible operation and the line of minimum-Q (related to reversible operation).

As a conclusion, any bithermal system $\mathrm{M}\left(q_{h}, q_{c}, w\right)$ can be interpreted graphically with the specific energy flow intensity related to the distance $r_{M}$ from the origin $\mathrm{M}^{\circ}(0,0,0)$ and its performance linked to the angle $\alpha$ only and bounded by $\alpha^{C}$. Thanks to the normed ternary diagram introduced in this paper, the graphical interpretation of bithermal systems is thus eased as it allows a direct visual comparison of the operating conditions of bithermal systems with the introduction of polar coordinates. This finding represents the main innovation compared to the representation of Raveau [9-11], as it allow a more intuitive graphical interpretation of bithermal systems operation.

\section{Illustrative Examples}

In order to illustrate the use of the proposed representation, bithermal systems operation of some exoreversible thermodynamics cycles (Carnot, Stirling, Erricson) using air perfect gas as working fluid are plotted in the proposed ternary diagram. Endoreversible (exoirreversible) bithermal systems operation in heat engine mode is also plotted on the graphical representation proposed in order to rediscover graphically the heat engine efficiency at maximum power revealed at the same time by Chambadal [14], Novikov [15] and Curzon-Ahlborn [16].

\subsection{Exoreversible Cycles}

This section is dedicated to plot the operation of the Carnot, Stirling and Erricson (exoreversible) cycles for Laplace perfect gas on the proposed ternary diagram. The thermodynamic cycles considered are depicted in Figure 7 in a T-s diagram.

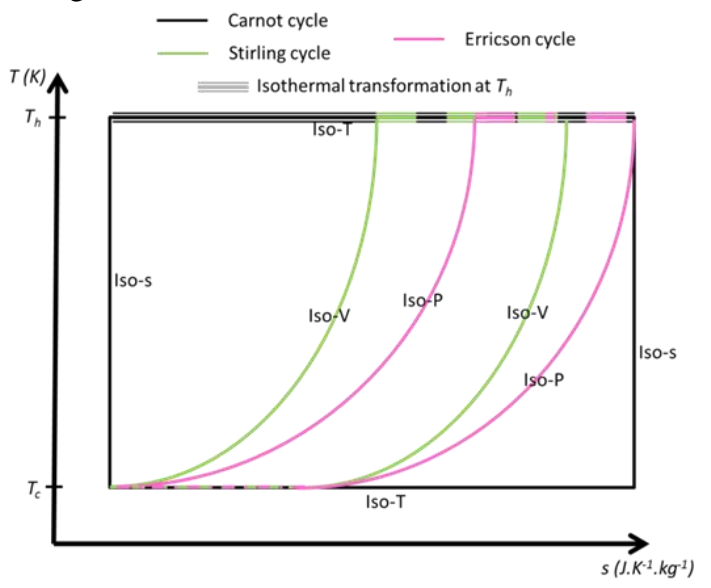

Figure 7. Carnot, Stirling and Erricson thermodynamic cycles plotted in T-s diagram (Figure is in color in the online version of the paper).

Following the Laplace theory, the specific heat capacities at constant pressure and constant volume of perfect gases express as follows:

$c_{P}=r \frac{\gamma}{\gamma-1}$ and $c_{V}=\frac{r}{\gamma-1}$

Where $r=R / M$ is the specific gas constant and $\gamma={ }^{C_{P}} / c_{V}$ is the isentropic expansion factor.

The energy balances over the cycles considered can be written as a function of the volume ratio $V^{*}=V_{q_{h}}^{i} / V_{q_{h}}^{f}$, defined as the ratio of the volumes at the initial and final states of the isothermal transformation at $T_{h}$ (highlighted with a triple line in Figure 7). The volume ratio $V^{*}$ is thus defined positive but could either be higher than unity in case of compression ( $w>0$, anti-clockwise cycle) or less than unity in case of expansion ( $w<0$, clockwise cycle).

According to the abundant literature on classical thermodynamics cycles [6-10], the specific energy balances over the cycles considered writes:

- Carnot cycle (two isothermal and two isentropic transformations)

$$
\begin{aligned}
& q_{h}{ }^{C}=-r T_{h} \ln \left(V^{*}\right) \\
& q_{c}{ }^{C}=r T_{c} \ln \left(V^{*}\right) \\
& w^{C}=r\left(T_{h}-T_{c}\right) \ln \left(V^{*}\right)
\end{aligned}
$$


- Stirling cycle without regenerator (two isothermal and two isovolume transformations)

Stirling cycle with perfect regenerator is strictly equivalent to a Carnot cycle [6-10]. Hence, Stirling cycle without regenerator is considered here. In this case, the specific energy balances over the cycle writes:

$$
\begin{aligned}
& q_{h}{ }^{S}=c_{v}\left(T_{h}-T_{c}\right)-r T_{h} \ln \left(V^{*}\right) \\
& q_{c}{ }^{S}=-c_{v}\left(T_{h}-T_{c}\right)+r T_{c} \ln \left(V^{*}\right) \\
& w^{S}=r\left(T_{h}-T_{c}\right) \ln \left(V^{*}\right)
\end{aligned}
$$

- Ericsson cycle (two isothermal and two isobaric transformations)

$$
\begin{aligned}
& q_{h}^{E}=c_{p}\left(T_{h}-T_{c}\right)-r T_{h} \ln \left(V^{*}\right) \\
& q_{c}{ }^{E}=-c_{p}\left(T_{h}-T_{c}\right)+r T_{c} \ln \left(V^{*}\right) \\
& w^{E}=r\left(T_{h}-T_{c}\right) \ln \left(V^{*}\right)
\end{aligned}
$$

The operating conditions of the considered thermodynamics cycles are plotted in the proposed ternary diagram with the volume ratio $V^{*}$ as parameter (Figure 8 for $\theta=1 / 3$ ). The different operating modes previously described are reached by each of the thermodynamic cycles considered depending on the volume ratio $V^{*}$. Obviously, any other bithermal thermodynamic cycles could also be plotted similarly in the proposed ternary diagram. The author restricts the analysis to the mentioned thermodynamic cycles only for sake of brevity.

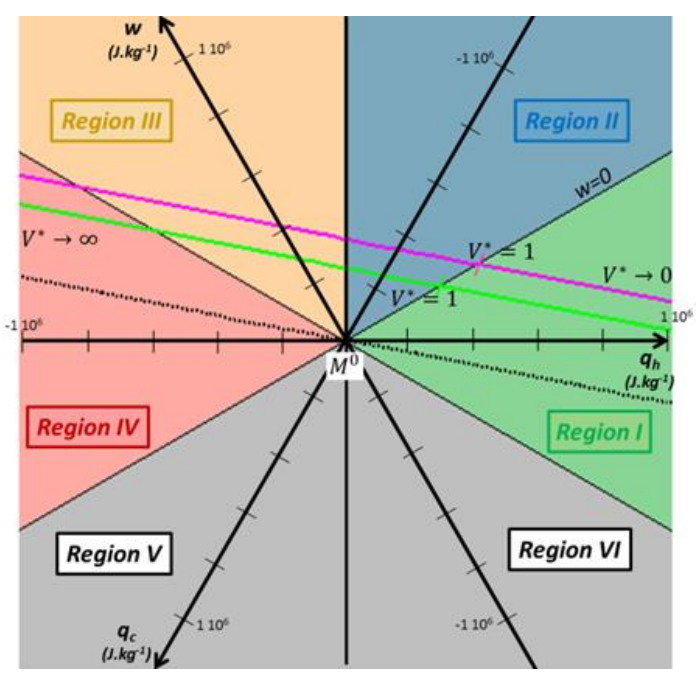

Figure 8. Operating conditions of bithermal thermodynamic systems for air perfect gas $(\gamma=1.4$ and $r=$ $\left.286.7 \mathrm{~J} . \mathrm{kg}^{-1} \cdot \mathrm{K}^{-1}\right)$ with $\theta=1 / 3$ - Carnot cycle: dotted black; Stirling cycle: green; Ericsson cycle: magenta (Figure is in color in the on-line version of the paper).

In agreement with the Carnot boundary defined in section 3.4, the Carnot cycle operation results in a straight line of angle $\alpha^{C}=1.761 \mathrm{rad}$ for $\theta=1 / 3$. Accordingly, the operation of the other irreversible thermodynamic cycles is restricted to the top half-plane.

For $V^{*}=1$, no work is exchanged with the system $(w=$ 0 , see Eqs. (22)-(24)). The corresponding bithermal cycle operations are thus plotted on the line perpendicular to the axis $\mathrm{w}(w=0$-axis). For the Carnot cycle, this particular operating condition corresponds to the origin $M^{\circ}(0,0,0)$ as no heat is exchanged with the system (thermodynamic equilibrium). However, when considering the Stirling and Erricson cycles, the heat exchanged during the isovolume and isobaric transformations respectively results in passive heat transfer $\left(q_{h}=-q_{c}\right.$, Region I/Region II interface).

The heat engine mode (Region I) is reached for $V^{*}<1$ for all the thermodynamic cycles considered. Under this condition, the cycle is operated clockwise resulting in generator operation, $w<0$.

Inversely, for $V^{*}>1$, bithermal cycles operate anticlockwise as receptor, $w>0$ (forced heat transfer, thermal dissipation or heat pump modes: Regions II, III and IV) depending on the volume ratio $V^{*}$. Low volume ratio $\left(\exp [-C / r(1-\theta)]>V^{*}>1\right)$ implies the Stirling and Erricson cycles to operate in forced heat transfer mode (Region II), as passive heat transfer (from the hot reservoir to the cold one) during the isovolume or isobaric transformations respectively predominate $\left(q_{h}>0\right.$ and $q_{c}<$ $0)$. Thermal dissipation mode (Region III) is reached for $\exp [-C / r \cdot(1 / \theta-1)]>V^{*}>\exp [-C / r \cdot(1-\theta)]$, when passive heat transfer is compensated by the isothermal transformation with the hot reservoir $\left(q_{h}<0\right)$. Finally, higher volume ratio $\left(V^{*}>\exp \left[-C / r \cdot\left(1 / t_{\theta}-1\right)\right]\right)$ is needed to operate the Stirling and Erricson cycles in heat pump mode (Region IV) to struggle the passive heat transfer during the isothermal transformation with the cold reservoir $\left(q_{c}>0\right)$.

As shown by Eqs. (22)-(24), the specific work over the Carnot, Stirling and Erricson cycles are identical, whereas the isovolume or isobaric transformations of the Stirling and Erricson cycles respectively results in an additional term for the hot and cold heat flows compared to the Carnot cycle. Consequently, Stirling and Erricson cycles operation are parallel to the Carnot cycle operation and the parametric curves are shifted along the $w=0$-axis because of this complementary term relative to passive heat transfer. Near reversible operation $\left(\alpha \rightarrow \alpha^{C}\right)$ could only be reached for $V^{*} \rightarrow 0$ (heat engine mode) or $V^{*} \rightarrow+\infty$ (heat pump mode), when passive heat transfer becomes negligible.

\subsection{Endoreversible Heat Engine Systems}

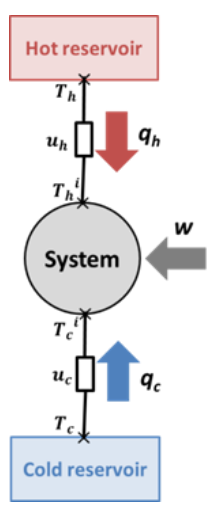

Figure 9. Endoreversible bithermal system (Figure is in color in the on-line version of the paper).

Let us now consider an endoreversible (exo-irreversible) bithermal system, as presented in Figure 9. The system follows the Carnot cycle operation with the intermediary temperatures $\mathrm{T}_{\mathrm{c}}{ }^{\mathrm{i}}$ and $T_{h}{ }^{i}$, which are linked to the reservoirs 
temperature $T_{c}$ and $T_{h}$ via the specific thermal conductances $u_{c}$ and $u_{h}$ such as:

$$
\begin{aligned}
& q_{h}=q_{h}{ }^{C}=u_{h}\left(T_{h}-T_{h}{ }^{i}\right) \\
& q_{c}=q_{c}{ }^{C}=u_{c}\left(T_{c}-{T^{c}}{ }^{i}\right)
\end{aligned}
$$

Similarly to previously, the operating conditions of the endoreversible bithermal systems are plotted with the volume ratio $V^{*}$ as parameter for different conductance values (with $u_{h}=u_{c}$ ) in Figure 10 .

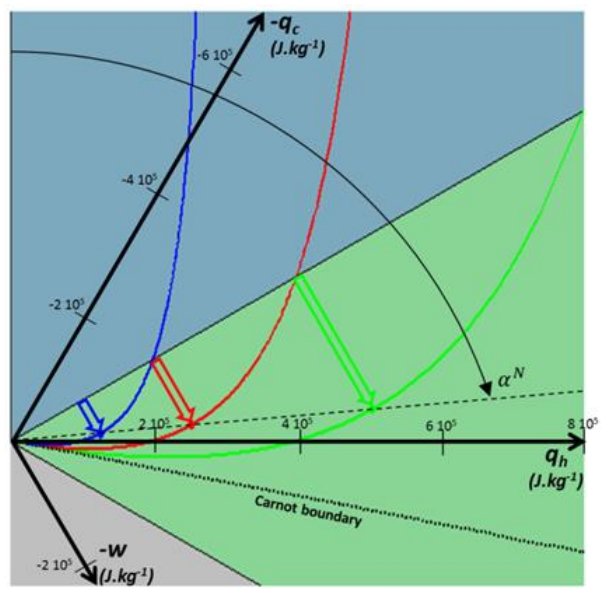

Figure 10. Endoreversible heat engine systems operation for air perfect gas $\left(\gamma=1.4\right.$ and $\left.r=286.7 \mathrm{~J} . \mathrm{kg}^{-1} . \mathrm{K}^{-1}\right)$ for $\theta=1 / 3$, for $u_{h}=u_{c}=2.510^{-4} \mathrm{~J} . \mathrm{kg}^{-1} . K^{-1}$ - blue $; u_{h}=$ $u_{c}=510^{-4} \mathrm{~J} \cdot \mathrm{kg}^{-1} \cdot \mathrm{K}^{-1} \quad$ - $\quad \mathrm{red} ; \quad u_{h}=u_{c}=$ $110^{-3} \mathrm{~J} \cdot \mathrm{kg}^{-1} \cdot \mathrm{K}^{-1}$ - green (Figure is in color in the on-line version of the paper).

For each conductance value, the maximum work reachable (in absolute value) by the system in heat engine mode is highlighted with a large arrow. Independently of the conductance values, the energy efficiency at maximum work in heat engine mode is given by [14-16]:

$\eta_{q_{h^{+}}{ }^{N}}=1-\sqrt{\theta}$

And the corresponding maximum work is:

$\operatorname{Max}\left|W_{<0}\right|=u_{h} T_{h} \frac{(1-\sqrt{\theta})^{2}}{1+{ }^{u_{h} / u_{c}}}$

These results are rediscovered graphically in Figure 10, as the operation conditions of maximum work are aligned on the dotted line of constant angle $\alpha^{N}$ defined by:

$\tan \alpha^{N}=\frac{\sqrt{3}}{1-2 \eta_{q_{h^{+}}}{ }^{N}}=\frac{\sqrt{3}}{2 \sqrt{\theta}-1}$

In the example plotted in Figure 10 for $\theta=1 / 3$, the

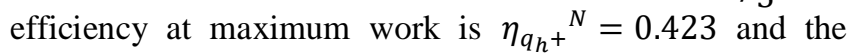
corresponding angle $\alpha^{N}$ is $1.482 \mathrm{rad}$. The corresponding maximum specific work (in absolute value) are $2.1410^{5}$, $1.0710^{5}$ and $5.3610^{4}{\mathrm{~J} . \mathrm{kg}^{-1}}^{-1}$ for the specific conductances equal to $10^{3}, 210^{3}$ and $410^{3} \mathrm{~J}_{\mathrm{kg}} \mathrm{kg}^{-1} \cdot \mathrm{K}^{-1}$, respectively.

\section{Conclusions}

This paper introduces an original and synthetic representation of bithermal thermodynamic system operation on a ternary diagram $\left(q_{h}, q_{c}, w\right)$. With respect to the energy flow directions, six different sectors (sextants) are identified and qualified: in addition to the two potentially reversible modes (heat engine and heat pump modes), two dissipative modes (forced heat transfer and thermal dissipation modes) are detailed. The two last sectors correspond to impossible operating modes, in accordance with the second law of thermodynamics. The qualification of the operating mode interfaces illustrates the physical continuity of the proposed representation.

Discussion on the energy efficiency definitions leads to the introduction of an unconventional definition when the hot reservoir operates as a sink $\left(q_{h}<0\right)$. This convenient definition results from the complementarity of systems operating in heat engine and heat pump modes. Accordingly, the energy efficiencies are kept positive and below unity for both reversible modes (heat engine and heat pump modes) and negative for both dissipative modes (thermal dissipation and forced heat transfer), leading to a mirror symmetry.

Following, the system operation is interpreted using polar coordinates. The energy intensity involved in the system (related to the specific energy flows) is linked to the distance $r_{M}$ of the system point $\mathrm{M}\left(q_{h}, q_{c}, w\right)$ to the origin $M^{\circ}(0,0,0)$ and its energy efficiency only depends on the angle $\alpha$ defined in section 5. Reversible operation consideration leads to the introduction of the Carnot boundary that restricts the operation of any bithermal system to the top half-plane bounded by the corresponding maximum angle $\alpha^{C}$ (function of the reservoirs temperature).

Finally, the use of the proposed representation is illustrated with two simple cases: (i) exoreversible thermodynamic cycles (Carnot, Stirling, Ericcson) are plotted in the ternary diagram with the compression volume ratio of the isothermal transformation at $T_{h}$ as parameter, that allows comparing the operating conditions of these cycles within the different operating modes; (ii) endoreversible (exo-irreversible) cycles are plotted to rediscover graphically the energy efficiency of Chambadal, Novikov and CurzonAhlborn at maximum work in heat engine mode $\left(\eta^{N}=1-\right.$ $\sqrt{\theta})$, as the corresponding operating points are aligned on a line of constant angle $\alpha^{N}$.

This original graphical representation may be highly advantageous for educational as well as research purposes, as it would help to compare and design bithermal systems with regards to the energies involved and its corresponding efficiency. Furthermore, the ternary diagram introduced herein could be generalized by analogy to thermochemical systems exchanging (thermal, potential, chemical or electrical...) energies with two reservoirs, one at low thermodynamic potential $\mu_{-}$and the other at high thermodynamic potential $\mu_{+}$.

\section{Acknowledgements}

The author would like to thank colleagues Prof. C. Ménézo, Prof. C. Ruyer-Quil, Assoc. Prof. N. Le Pierrès and Ph.D. M. Perriet-Muzet, along with Prof. C. Goupil (Université ParisDiderot, France) for enlightening discussions and constructive feedback on the manuscript. The graphical contribution of Ph.D. student N. Cellier is also acknowledged. This research received no specific grant from any funding agency in the public, commercial, or not-for-profit sectors.

\section{Nomenclature}

$c_{P} \quad$ Specific heat capacity at constant pressure, $\mathrm{J} \cdot \mathrm{kg}^{-1} \cdot \mathrm{K}^{-1}$

$\mathrm{c}_{\mathrm{V}} \quad$ Specific heat capacity at constant volume, $\mathrm{J} \cdot \mathrm{kg}^{-1} \cdot \mathrm{K}^{-1}$

COP Coefficient of performance, -

M Molar mass, $\mathrm{kg} \cdot \mathrm{mol}^{-1}$

q Specific heat energy, $\mathrm{J} \cdot \mathrm{kg}^{-1}$ 
$\mathrm{r}_{\mathrm{M}} \quad$ Radius, $\mathrm{J} \cdot \mathrm{kg}^{-1}$

r Specific gas constant, $\mathrm{J} \cdot \mathrm{kg}^{-1} \cdot \mathrm{K}^{-1}$

$R \quad$ Perfect gas constant, $\mathrm{J} \cdot \mathrm{mol}^{-1} \cdot \mathrm{K}^{-1}$

$T$ Temperature, $\mathrm{K}$

$\mathrm{u} \quad$ Specific Thermal conductance, $\mathrm{J} \cdot \mathrm{kg}^{-1} \cdot \mathrm{K}^{-1}$

$\mathrm{V}^{*}$ Volume ratio, -

w Specific work, $\mathrm{J} \cdot \mathrm{kg}^{-1}$

\section{Greek symbols}

$\alpha \quad$ Angle, rad

$\Delta \mathrm{T} \quad$ Temperature difference, $\mathrm{K}$

$\gamma \quad$ Isentropic expansion factor, -

$\theta \quad$ Reservoirs temperature ratio, -

$\eta \quad$ Energy efficiency, -

$\sigma \quad$ Specific entropy generation, $\mathrm{J} \cdot \mathrm{kg}^{-1} \cdot \mathrm{K}^{-1}$

\section{Superscripts and subscripts}

C Carnot cycle

Cold reservoir

E Erricson cycle

$h$ Hot reservoir

$m \quad$ Equivalent monothermal system

N Novikov (/Chambadal/Curzon-Ahlborn) system

qc+ Hot heat source

qc- Hot heat sink

s Stirling cycle

\section{References:}

[1] S. Carnot, Réflexions sur la puissance motrice du feu et sur les machines propres à développer cette puissance. Paris: Bachelier, 1824. (transl. Carnot NLS, Thurston $\mathrm{RH}$, Reflections on the motive power of heat, and on machines fitted to develop that power. New York: John Wiley \& Sons, 1897).

[2] R. Clausius, "Ueber die bewegende Kraft der Wärme und die Gesetze, welche sich daraus für die Wärmelehre selbst ableiten lassen", Annalen der Physik, 79: 368-397, 500-524, 1850. (transl. "On the moving force of heat, and the laws regarding the nature of heat itself which are deducible therefrom", Phil. Mag. 2, 102-119, 1851).

[3] J.P. Joule, "On the mechanical equivalent of heat", Phil. Trans. R. Soc. 140, 61-82, 1850.

[4] E. Clapeyron, "Mémoire sur la puissance motrice de la chaleur", Journal de l'École Royale Polytechnique, 14:
23, 153-190, 1834. (transl. E. Clapeyron and R. Clausius, Memoir on the motive power of heat, in Reflections on the motive power of fire by S. Carnot and other papers on the second law of thermodynamics, Mineola: NY Dover Publications, 1960).

[5] R. Mollier, Neue Diagramme zur Technischen Wärmelehre, Berlin, 1904. (transl. New Graphs for Technical Thermodynamics).

[6] A. Bejan, Advanced Engineering Thermodynamics, $4^{\text {th }}$ Ed. Hoboken: Wiley, 2016.

[7] M. Moran, H. Shapiro, Fundamentals of engineering thermodynamics, $6^{\text {th }} E d$. USA: John Wiley \& Sons, Inc., 2008.

[8] L. Borel, D. Favrat, Thermodynamics and Energy Systems Analysis: From Energy to Exergy, Vol. 1. Lausanne: EPFL Press, 2010.

[9] J.-P. Pérez, Thermodynamique : Fondements et applications, Vol. 1, $3^{\text {rd }}$ Ed. Paris: Dunod, 2001. [in French]

[10]C. Lhuillier, J. Rous, Introduction à la thermodynamique. Paris: Dunod, 1992. [in French]

[11] Wikipedia Diagramme de Raveau. Available: https://fr.wikipedia.org/wiki/Diagramme_de_Raveau (accessed Sep. 25, 2017) [in French].

[12] G. Alefeld, R. Radermacher. Heat Conversion Systems. Boca Raton: CRC Press, 1993.

[13] J. Ramousse, "Représentation graphique des modes opératoires des systèmes dithermes". in SFT 2016, Proceedings of congrès annuel de la Société Française de Thermique, Toulouse, 31 May - 3 June 2016. [in French]

[14] P. Chambadal, Les centrales nucléaires. Paris : Armand Colin, 1957. [in French]

[15] I.I. Novikov, "The efficiency of atomic power stations (a review)". J. Nucl. Energy II, 7, 125-128, 1958.

[16] F. L. Curzon, B. Ahlborn, "Efficiency of a Carnot engine at maximum power output". Am. J. Phys., 43, 2224, 1975. 